\title{
L'entrevue motivationnelle : un outil particulièrement efficace pour atténuer la réticence à la vaccination
}

\author{
Arnaud Gagneur ${ }^{1,2 \star}$
}

\section{Résumé}

L'Organisation mondiale de la santé classe la réticence à la vaccination parmi les dix principales menaces pour la santé mondiale. Les stratégies capables de lutter efficacement contre ce problème croissant sont peu nombreuses. Au Canada, environ $20 \%$ des parents et aidants émettent des réserves quant à la vaccination de leur enfant. Tenter de les convaincre en leur présentant simplement des faits sur la vaccination pourrait se révéler contreproductif et même accentuer leur réticence. Comment, dans ce contexte, les fournisseurs de soins peuvent-ils surmonter la difficulté de répondre aux besoins des parents amenés à prendre des décisions quant à la vaccination de leur enfant?

L'entrevue motivationnelle a pour objectif de faciliter le processus décisionnel en recueillant et renforçant les motivations d'une personne à changer de comportement à partir de ses propres arguments en faveur du changement. Cette approche s'articule autour de trois composantes principales : la volonté d'instaurer une culture axée sur la collaboration et la compassion; les processus destinés à favoriser l'engagement dans la relation et à orienter la conversation autour de l'objectif du changement; et les compétences dont les fournisseurs de soins ont besoin pour comprendre les véritables préoccupations du parent ou de l'aidant et y répondre.

En ce qui concerne la vaccination, la technique d'entrevue motivationnelle vise à renseigner les parents ou aidants au sujet de la vaccination en tenant compte de leurs besoins particuliers et de leur niveau individuel de connaissances, et en respectant leurs convictions. Le recours à l'entrevue motivationnelle prend la forme d'une conversation respectueuse et empathique autour de la vaccination et contribue à nouer une relation solide.

De nombreuses études menées au Canada, y compris des essais cliniques randomisés multicentriques, ont démontré l'efficacité de la technique d'entrevue motivationnelle. Depuis 2018, la stratégie PromoVac, une intervention de sensibilisation fondée sur la technique d'entrevue motivationnelle, a été mise en oeuvre à titre de nouvelle pratique de soins dans les maternités du Québec, dans le cadre du Programme d'entretien motivationnel en maternité pour l'immunisation des enfants (EMMIE).
Cette oeuvre est mise à la disposition selon les termes de la licence internationale Creative Commons Attribution 4.0

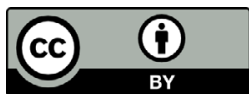

Affiliations

1 Département de pédiatrie, Université de Sherbrooke, Sherbrooke, QC

${ }^{2}$ Centre de recherche du Centre hospitalier universitaire de Sherbrooke, Sherbrooke, QC

\section{*Correspondance :}

arnaud.gagneur@usherbrooke.ca

Citation proposée : Gagneur A. L'entrevue motivationnelle : un outil particulièrement efficace pour atténuer la réticence à la vaccination. Relevé des maladies transmissibles au Canada 2020;46(4):104-9

https://doi.org/10.14745/ccdr.v46i04a06f

Mots-clés : réticence à la vaccination, entrevue motivationnelle, acceptation de la vaccination, recours à la vaccination, préoccupations parentales

\section{Introduction}

Tenter de convaincre des parents ou aidants réticents à la vaccination de faire vacciner leur enfant en leur présentant simplement des faits sur la vaccination pourrait se révéler contreproductif et même accentuer leur réticence (1). Dans une revue systématique Cochrane, Kaufman et coll. ont conclu qu'une intervention en personne exclusivement axée sur la communication de renseignements pratiques et logistiques sur la vaccination avait peu de chance d'être fructueuse si elle ne tient pas compte des convictions des parents (2). Néanmoins, dans une autre revue systématique Cochrane évaluant les perceptions et les expériences des parents et des aidants à l'égard de la communication sur la vaccination systématique des enfants, les 
auteurs ont constaté que les parents désiraient recevoir plus de renseignements que ceux qui leur étaient communiqués, et souhaitaient que ces renseignements soient factuels, simples, contextuels et présentés en temps opportun par un fournisseur de soins digne de confiance (3).

Le message à retenir de la littérature est que les parents et aidants désirent recevoir plus de renseignements, mais que les méthodes de sensibilisation traditionnelles ne répondent pas à leurs besoins. Cela étant, comment devrait-on présenter aux parents les faits concernant la vaccination? Cette question est d'autant plus cruciale que l'Organisation mondiale de la santé a classé la réticence à la vaccination parmi les dix principales menaces pour la santé mondiale (4). Jusqu'à récemment, peu de stratégies se sont révélées capables de lutter efficacement contre le problème croissant de la réticence à la vaccination (c'est-à-dire la méfiance envers la vaccination ou le refus de se faire vacciner malgré l'accessibilité des vaccins) (1).

La technique d'entrevue motivationnelle (EM) est l'une des rares stratégies ayant contribué à améliorer la couverture vaccinale des nourrissons et à réduire la réticence à la vaccination des parents (5-10). L'EM est une méthode de communication centrée sur la personne dont le but est de renforcer sa motivation à changer de comportement en pointant son ambivalence intrinsèque et en la dépassant (11). C'est une technique jugée prometteuse pour la promotion de la santé (12), et le Comité consultatif national de l'immunisation (CCNI) en recommande actuellement l'utilisation dans le contexte de la vaccination (13).

Les données étayant l'efficacité de l'EM sur la réticence à la vaccination sont convaincantes. Gagneur et d'autres chercheurs ont mis au point une intervention de sensibilisation fondée sur la technique d'EM à l'intention des parents séjournant à la maternité après l'accouchement (stratégie PromoVac) (5-8). Une étude pilote régionale a montré que cette stratégie s'était traduite par une augmentation de $15 \%$ de l'intention de vaccination des mères, une hausse de $7 \%$ de la couverture vaccinale des nourrissons à sept mois, et par une probabilité accrue de $9 \%$ que les enfants présentent un statut vaccinal complet à l'âge de deux ans si leurs parents recevaient I'intervention à la maternité (6-8). Un essai clinique randomisé mené à l'échelle provinciale a montré que les taux de réticence à la vaccination avaient chuté de $40 \%(5,6)$. Les mères réticentes à la vaccination sont celles pour lesquelles l'intervention a été la plus bénéfique, $97 \%$ d'entre elles s'en déclarant satisfaites et prêtes à la recommander à tous les parents (7).

Dempsey et coll. ont également démontré l'efficacité d'une stratégie de promotion de la vaccination se fondant sur l'EM pour accroître le recours à la vaccination contre le virus du papillome humain (VPH) chez les adolescents (10).
En 2018, la stratégie PromoVac a été mise en œuvre dans toutes les maternités du Québec dans le cadre d'un programme provincial de santé publique intitulé «EMMIE » (Entretien motivationnel en maternité pour l'immunisation des enfants). Avec une hausse de $11 \%$ de l'intention de vaccination et une baisse de $30 \%$ du taux de réticence à la vaccination, les résultats préliminaires de l'évaluation de la stratégie PromoVac ont permis de corroborer ceux des études précédentes (14).

Cet article vise à définir la technique d'entrevue motivationnelle et à illustrer son utilité dans la lutte contre la réticence à la vaccination. Il met en lumière son impact sur la réticence à la vaccination et la couverture vaccinale, et décrit son utilisation dans le cadre d'un programme de santé publique mis en œuvre dans les maternités du Québec (12).

Il s'agit du troisième d'une série d'articles produits par le Centre canadien de ressources et d'échange sur les données probantes en vaccination (CANVax) (15). Ce centre accueille une équipe pluridisciplinaire de professionnels chargés de répertorier et créer des ressources utiles dans le but de favoriser le recours à la vaccination (16).

\section{Pratiques exemplaires en matière d'entrevue motivationnelle}

L'EM est une technique d'entrevue ayant pour but de renforcer la motivation et l'engagement de la personne interrogée. Pour le professionnel de la santé, il ne s'agit pas tant de discuter avec le parent ou l'aidant ou de lui dire quoi faire, mais plutôt de collaborer avec lui. L'EM vise à renforcer la motivation et l'engagement d'une personne à l'égard d'un objectif particulier en recueillant et décryptant ses raisons personnelles en faveur du changement, et ce, dans un esprit d'acceptation et de compassion (11).

L'EM s'articule autour de trois composantes principales : L'esprit ou la volonté d'instaurer une relation axée sur la collaboration et la compassion; les processus destinés à favoriser l'engagement dans la relation et à orienter la conversation autour de l'objectif du changement; et les outils nécessaires aux fournisseurs de soins pour comprendre les véritables préoccupations du parent ou de l'aidant et y répondre.

\section{Instaurer une culture axée sur la collaboration et la compassion}

Les fournisseurs de soins s'appuient sur les quatre éléments de l'esprit de l'EM pour tisser une relation empreinte de respect et de compassion :

- Partenariat - Rapport d'égal à égal, faciliter l'échange

- Acceptation - Adopter une attitude positive et empathique pour renforcer l'autonomie

- Évocation - Amener la personne à verbaliser le changement

- Compassion/Altruisme - Agir avec bienveillance 


\section{Favoriser l'engagement dans la relation et cibler l'objectif de l'intervention}

L'EM prévoit quatre processus successifs pour faciliter l'engagement dans la relation avec le parent ou l'aidant et l'orienter vers un objectif de changement adapté en fonction de ses capacités (tableau 1).

Tableau 1 : Les quatre processus successifs de l'entrevue motivationnelle

\begin{tabular}{|l|l|l|}
\hline Processus & \multicolumn{1}{|c|}{ Objectifs } & Questions à poser \\
\hline Engagement & $\begin{array}{l}\text { Renforcer le lien, } \\
\text { témoigner sa compassion } \\
\text { et son intérêt }\end{array}$ & $\begin{array}{l}\text { Quelle est la réalité de } \\
\text { la personne? }\end{array}$ \\
\hline Écalisation & $\begin{array}{l}\text { Définir l'objectif de } \\
\text { changement et orienter la } \\
\text { conversation autour de cet } \\
\text { objectif }\end{array}$ & $\begin{array}{l}\text { Quel est l'objectif } \\
\text { de changement à } \\
\text { poursuivre? }\end{array}$ \\
\hline Évocation & $\begin{array}{l}\text { Objectif } 1: \text { Raisons du } \\
\text { changement et capacités } \\
\text { à changer (importance du } \\
\text { changement) } \\
\text { Objectif 2: Le discours } \\
\text { changement (confiance à } \\
\text { l'égard du changement) }\end{array}$ & $\begin{array}{l}\text { Quel serait l'intérêt } \\
\text { d'évoluer vers le } \\
\text { changement? } \\
\text { Quels sont les } \\
\text { compétences et } \\
\text { les points forts que } \\
\text { possède la personne } \\
\text { pour y parvenir? }\end{array}$ \\
\hline Planification & $\begin{array}{l}\text { Discussion sur } \\
\text { l'engagement. Comment } \\
\text { changer? }\end{array}$ & $\begin{array}{l}\text { Comment la personne } \\
\text { y parviendra-t-elle? }\end{array}$ \\
\hline
\end{tabular}

Ces processus ne sont pas linéaires, et ne constituent pas un guide par étape sur l'EM. L'engagement dans la relation arrive en premier, car il est requis avant de pouvoir entamer une conversation sur le changement. Si à tout moment la personne se désengage de la relation, le fournisseur de soins doit retourner à l'étape de l'engagement pour réengager la relation.

\section{Comprendre le parent ou l'aidant et s'adapter à ses besoins particuliers}

Le fournisseur de soins cerne et comprend les véritables préoccupations du parent ou de l'aidant, et peut, grâce I'utilisation des outils spécifiques de l'EM renforcer sa motivation en faveur du changement. Les outils de l'EM comprennent, entre autres, l'utilisation de questions ouvertes, la pratique de l'écoute réflexive ainsi que la valorisation et le résumé des propos de la personne (tableau 2). Ces compétences s'inscrivent dans le cadre d'une dynamique pendant laquelle le fournisseur de soins écoute activement le parent ou l'aidant, puis reformule ses propos tout en soulignant les points positifs de son comportement. Cela peut contribuer à un regain de confiance du parent ou de l'aidant à l'égard du changement.
Tableau 2 : Compétences en entrevue motivationnelle

\begin{tabular}{|c|c|c|}
\hline Compétences & Objectifs & Exemples \\
\hline $\begin{array}{l}\text { Questions } \\
\text { ouvertes }\end{array}$ & $\begin{array}{l}\text { Susciter des réponses et } \\
\text { éviter les doutes }\end{array}$ & $\begin{array}{l}\text { Questions ouvertes : } \\
\text { ("Qu'avez-vous } \\
\text { compris? »/«Qu'en } \\
\text { pensez-vous? ») } \\
\text { Questions fermées : } \\
\text { ("Avez-vous } \\
\text { compris? »/« Croyez-vous } \\
\text { que c'est important? ») }\end{array}$ \\
\hline Valorisation & $\begin{array}{l}\text { Encourager la personne } \\
\text { et mettre en évidence ses } \\
\text { points forts }\end{array}$ & $\begin{array}{l}\text { "La santé et la sécurité de } \\
\text { vos enfants occupent une } \\
\text { grande importance à vos } \\
\text { yeux. " } \\
\text { "Vous savez déjà } \\
\text { beaucoup de choses. » }\end{array}$ \\
\hline $\begin{array}{l}\text { Écoute réflexive } \\
\text { et résumés }\end{array}$ & $\begin{array}{l}\text { Permettre à la personne } \\
\text { de nuancer et de rectifier } \\
\text { ses propos } \\
\text { Reflet simple : ce que la } \\
\text { personne dit } \\
\text { Reflet complexe : ce } \\
\text { qu'elle veut dire }\end{array}$ & $\begin{array}{l}\text { «Vous avez lu des articles } \\
\text { sur la relation entre les } \\
\text { vaccins et les troubles } \\
\text { comme l'autisme " "Ce } \\
\text { qui vous importe le plus, } \\
\text { c'est que votre enfant } \\
\text { jouisse du meilleur état de } \\
\text { santé possible. " }\end{array}$ \\
\hline $\begin{array}{l}\text { Comment } \\
\text { fournir des } \\
\text { renseignements } \\
\text { ou des conseils: }\end{array}$ & $\begin{array}{l}\text { Comment fournir des } \\
\text { renseignements ou des } \\
\text { conseils : } \\
\text { DEMANDER = demander } \\
\text { au parent ou à l'aidant } \\
\text { ce qu'il sait, et lui } \\
\text { demander la permission } \\
\text { de compléter ses } \\
\text { connaissances } \\
\text { PARTAGER = fournir des } \\
\text { renseignements ou des } \\
\text { conseils sur le sujet } \\
\text { DEMANDER = vérifier } \\
\text { si le parent ou l'aidant a } \\
\text { compris l'information et ce } \\
\text { qu'il compte faire avec }\end{array}$ & $\begin{array}{l}\text { «Si vous êtes d'accord, je } \\
\text { pourrais compléter... " } \\
\text { "Quel sens donnez-vous à } \\
\text { cette nouvelle } \\
\text { information?» }\end{array}$ \\
\hline
\end{tabular}

\section{Pourquoi l'entrevue motivationnelle fonctionne avec un parent ou un aidant réticent à la vaccination}

Le recours à l'EM lors d'une séance d'information contribue à tisser une relation orientée sur le parent ou l'aidant et, fait important, à accueillir les parents à leur niveau individuel de connaissances et dans le respect de leurs convictions (5-8). Le recours à la technique d'EM prend la forme d'une conversation respectueuse et empathique autour de la vaccination et contribue à tisser une relation solide entre le parent ou l'aidant et le professionnel de la santé (9). Les parents sont libres d'exprimer leurs préoccupations et de poser des questions sur la vaccination sans crainte d'être jugés $(6,9)$. Les professionnels de la santé peuvent cerner et cibler les préoccupations et les idées fausses des parents sur la vaccination et leur communiquer des renseignements personnalisés sur le sujet (6). 
Le ciblage des préoccupations et la personnalisation des renseignements sont les principales caractéristiques qui distinguent cette technique des autres interventions actuelles dans le domaine de la promotion de la vaccination. Ces particularités pourraient expliquer les résultats particulièrement positifs obtenus avec les technique d'EM pour diminuer la réticence à la vaccination et améliorer les couvertures vaccinales (5-8). La séance d'information avec EM est tout à fait adaptée pour répondre aux besoins, aux préoccupations et aux questions des parents ou des aidants en ce qui concerne la vaccination de leur enfant. Grâce aux techniques d'EM, les professionnels de la santé aident les personnes à mettre en lumière leur propre ambivalence, à trouver leurs propres arguments en faveur du changement et à décider par elles-mêmes, en toute connaissance de cause, de faire vacciner ou non leur enfant. Dans une étude relative au processus décisionnel des parents ou aidants en matière de vaccination, Paulussen et coll. ont montré que la plupart des parents ou aidants n'étudiaient pas activement les renseignements qui leur étaient fournis sur les avantages et les inconvénients avant de décider de faire vacciner ou non leur enfant (17). L'attitude d'un parent à l'égard de la vaccination et son intention de faire vacciner son enfant sont ainsi susceptibles d'évoluer face à des contre-arguments étayés ou non. En recueillant et décryptant les raisons personnelles pour lesquelles un parent choisit la vaccination, la technique d'EM renforce sa motivation personnelle à recourir à la vaccination grâce à un processus décisionnel robuste. Par ailleurs, l'EM est une intervention de courte durée qui pourrait facilement être intégrée à la consultation habituelle de vaccination une fois les professionnels de la santé formés à sa pratique.

Le tableau 3 illustre la manière dont se déroulerait une conversation sur la vaccination entre un parent et un fournisseur de soins si on appliquait, d'une part, la méthode classique, et d'une part, la technique d'entrevue motivationnelle.

\section{Tableau 3 : Exemple illustrant l'application de la méthode classique et de la technique d'entrevue motivationnelle à une conversation sur la vaccination}

\section{Méthode classique fondée \\ sur l'information et le} counseling

FDS : Il est important que votre enfant soit vacciné. Sinon, vous risquez de le mettre en danger. Saviez-vous qu'il y a encore des cas de rougeole au Canada? C'est une maladie qui peut être très dangereuse. Et la méningite? On peut en mourir, vous savez. Vous devriez mettre les vaccins de votre enfant à jour, car il est déjà en retard dans le calendrier. Nous pouvons le faire maintenant si vous voulez.

Mère : Je ne vois pas où est

l'urgence. Et l'autisme est bien pire que la rougeole! Ce vaccin apporte plus de problèmes que de solutions. En plus, c'est vraiment incroyable de donner autant de vaccins en même temps! j'hésite quand même.

Tableau 3 : Exemple illustrant l'application de la méthode classique et de la technique d'entrevue motivationnelle à une conversation sur la vaccination (suite)

\section{Technique d'entrevue} motivationnelle

\section{Méthode classique fondée sur l'information et le counseling}

FDS : Des études ont montré qu'il n'y avait aucun lien entre l'autisme et le vaccin contre la rougeole. Le vaccin est sûr, croyez-moi. Il faut faire attention aux informations que l'on trouve sur Internet. II n'y a aucun danger à faire plusieurs vaccins en même temps, et ce n'est pas plus douloureux. II vaudrait mieux mettre ses vaccins à jour maintenant.

Mère : J'ai entendu le contraire, et pas seulement sur Internet. Je me suis beaucoup renseigné sur le sujet, et la vaccination n'est pas obligatoire, rien ne m'oblige à la faire.

FDS : Vous avez raison, ce n'est pas obligatoire, mais vous mettez en danger votre enfant ainsi que les autres enfants qui ne peuvent pas recevoir le vaccin. Les risques des maladies sont bien plus élevés que ceux des vaccins. $\mathrm{Si}$ je prends le temps de vous dire tout ça, c'est parce que c'est très important.

Mère : C'est facile pour vous! Vite, vite! Mais s'il devient autiste? Je $m$ 'inquiète du risque des vaccins, mais vous n'avez pas l'air de vous inquiéter de la santé de mon fils.

FDS : Bien sûr que si! Et je m'inquiète du fait qu'il pourrait attraper des maladies qui sont évitables par la vaccination.

Mère : Je crois qu'on ne se comprend pas. Remettons cette conversation à une autre fois.

FDS : Que pensez-vous des avantages de la vaccination? [Question ouverte]

Mère : Eh bien, je sais que les vaccins protègent les enfants contre plusieurs maladies qu'on ne voit plus. Mon enfant a fait tous ses premiers vaccins, mais j'ai peur que celui contre la rougeole puisse le rendre autiste. Pour les autres vaccins, j'ai moins de doutes, mais
Résumé :

Le FDS a endossé un rôle de spécialiste et mené une intervention directive fondée sur l'argumentation et le réflexe correcteur. Ce type d'intervention a mené à une opposition.
Technique d'entrevue motivationnelle
FDS : Comme vous le dites, les vaccins ont fait tellement diminuer les maladies qu'elles sont maintenant beaucoup moins fréquentes. C'est pour cela que vous avez fait vacciner votre enfant quand il était bébé. Si je comprends bien, sauf pour le vaccin contre la rougeole, les autres vaccins vous paraissent sûrs. [Résumé; reflet complexe]

Mère : Oui, je sais qu'il est important de prévenir ces infections. Mais pour la rougeole, je suis partagée. J'ai lu beaucoup de livres et d'articles, vous savez. Beaucoup de gens s'inquiètent du lien entre le vaccin contre la rougeole et l'autisme.

FDS : Si je comprends bien, vous pensez qu'il est important de protéger votre enfant contre les maladies quand les vaccins sont sûrs, mais vous vous inquiétez à cause de ce que vous avez entendu dire sur l'autisme et le vaccin contre la rougeole. [Résumé] Je vois que vous avez fait beaucoup de recherches sur le sujet. [Valorisation] Si vous le souhaitez, je pourrais vous donner des informations supplémentaires sur l'autisme et la rougeole. [Demander]

Mère : Avec plaisir! Je veux savoir exactement ce qu'il en est.

FDS : En fait, vous avez raison. Une étude avait posé I'hypothèse d'un lien entre le vaccin contre la rougeole et l'autisme, mais cette étude était fausse et l'auteur a perdu sa licence médicale. Plus de 500 autres études réalisées partout dans le monde ont montré qu'il n'y avait aucun lien entre ce vaccin et l'autisme. La fréquence de l'autisme est la même chez les enfants vaccinés et non vaccinés. [Partager] Qu'en pensez-vous? [Demander, dernière étape de Demander-Partager-Demander]

Mère : Bien, alors je ne suis pas folle de $m$ 'inquiéter de ça?

FDS : Bien sûr que vous, vous avez parfaitement raison. [Valorisation]

Mère : Merci d'avoir pris le temps de comprendre ce qui me préoccupe. Je pense que c'est un peu plus clair maintenant.

\section{Résumé :}

L'EM a permis à la mère d'exprimer ses préoccupations et son ambivalence sans être jugée. La méthode

Partager-Demander-Partager a permis au FDS de fournir à la mère des renseignements qu'elle a sollicités et qu'elle était prête à recevoir. 


\section{Conclusion}

L'EM est un outil particulièrement efficace pour améliorer l'acceptation de la vaccination et atténuer l'hésitation vaccinale $(18,19)$. Il s'agit d'une nouvelle pratique exemplaire que l'Organisation mondiale de la santé recommande d'intégrer à la formation des vaccinateurs et des fournisseurs de soins assurant la prestation de conseils sur la vaccination (20). Les nombreuses ressources de formation théorique ou pratique disponibles concernant l'utilisation de la technique d'entrevue motivationnelle dans le secteur de la vaccination s'avéreraient particulièrement utiles aux fournisseurs de soins qui souhaitent intégrer cette technique dans leur pratique quotidienne (18-22).

\section{Déclaration de l'auteur}

A. G. - Rédaction, révision et édition du manuscrit

\section{Conflit d'intérêts}

A. Gagneur a bénéficié de subventions de l'Agence de la santé publique du Canada, du ministère de la Santé et des Services sociaux du Québec, du Fonds de recherche du Québec - Santé, des Instituts de recherche en santé du Canada et du Réseau canadien de recherche sur l'immunisation.

\section{Remerciements}

Les contributions au Centre canadien de ressources et d'échange sur les données probantes en vaccination (CANVax) proviennent d'un très large éventail d'auteurs, de comités, de partenaires de vaccination, d'examinateurs et, surtout, du secrétariat du CANVax à l'Association canadienne de santé publique.

\section{Financement}

Les études PromoVac ont été financées par le Fonds de recherche du Québec - Santé ( $n^{\circ} 27505$ ). Ministère de la Santé et des Services sociaux du Québec, Instituts de recherche en santé du Canada et Réseau canadien de recherche sur l'immunisation.

\section{Références}

1. Dubé E, Gagnon D, MacDonald NE; SAGE Working Group on Vaccine Hesitancy. Strategies intended to address vaccine hesitancy: review of published reviews. Vaccine 2015 Aug;33(34):4191-203. DOl PubMed

2. Kaufman J, Ryan R, Walsh L, Horey D, Leask J, Robinson P, Hill S. Face-to-face interventions for informing or educating parents about early childhood vaccination. Cochrane Database Syst Rev 2018 May;5:CD010038. DOI PubMed
3. Ames HM, Glenton C, Lewin S. Parents' and informal caregivers' views and experiences of communication about routine childhood vaccination: a synthesis of qualitative evidence. Cochrane Database Syst Rev 2017 Feb;2:CD011787. DOI PubMed

4. Dix ennemis que l'OMS devra affronter cette année. Genève $(\mathrm{CH})$ : Organisation mondiale de la Santé; 2019. https://www. who.int/fr/news-room/feature-stories/ten-threats-to-globalhealth-in-2019

5. Gagneur A, Battista MC, Boucher F, Tapiero B, Quach C, De Wals $P$, Lemaître T, Farrands A, Boulianne N, Sauvageau C, Ouakki M, Gosselin V, Petit G, Jacques MC, Dubé E. Promoting vaccination at maternity wards using a motivational interview reduces hesitancy and enhances intention to vaccinate: a multicenter pre- and post-design RCT-nested study, Quebec, March 2014 to February 2015. Euro Surveill 2019;24(36): DOI PubMed

6. Gagneur A, Gosselin V, Dubé È. Motivational interviewing: A promising tool to address vaccine hesitancy. Vaccine 2018 Oct;36(44):6553-5. DOl PubMed

7. Gagneur A, Lemaître T, Gosselin V, Farrands A, Carrier N, Petit $G$, Valiquette $L$, De Wals $P$. A postpartum vaccination promotion intervention using motivational interviewing techniques improves short-term vaccine coverage: PromoVac study. BMC Public Health 2018 Jun;18(1):811. DOI PubMed

8. Lemaître T, Carrier N, Farrands A, Gosselin V, Petit G, Gagneur A. Impact of a vaccination promotion intervention using motivational interview techniques on long-term vaccine coverage: the PromoVac strategy. Hum Vaccin Immunother 2019;15(3):732-9. DOl PubMed

9. Boodman E. Special report: The vaccine whisperers: counselors gently engage new parents before their doubts harden into certainty. Boston (MA): Stat; 2019 Aug 5. https:// www.statnews.com/2019/08/05/the-vaccine-whispererscounselors-gently-engage-new-parents-before-their-doubtsharden-into-certainty/

10. Dempsey AF, Pyrznawoski J, Lockhart S, Barnard J, Campagna EJ, Garrett K, Fisher A, Dickinson LM, O'Leary ST. Effect of a health care professional communication training intervention on adolescent human papillomavirus vaccination: a cluster randomized clinical trial. JAMA Pediatr 2018 May; 172(5):e180016. DOI PubMed

11. Rollnick S, Miller WR, Butler CC. Motivational interviewing in health care: helping patients change behavior. New York: The Guilford Press; 2008.

12. Appiah-Brempong E, Okyere P, Owusu-Addo E, Cross R. Motivational interviewing interventions and alcohol abuse among college students: a systematic review. Am J Health Promot 2014 Sep-Oct;29(1):e32-42. DOI PubMed

13. Guide canadien d'immunisation : Partie 1 - Information clé sur l'immunisation. Ottawa (ON) : Gouvernement du Canada; (Accédé 2020-02-17). https://www.canada.ca/fr/santepublique/services/publications/vie-saine/guide-canadienimmunisation-partie-1-information-cle-immunisation.html 
14. Gagneur A, Bergeron J, Gosselin V, Guay M, Dubé Ė, Sanson J, Sicard N, Auger D. Preliminary results of EMMIE program: Increase of parental vaccine intention and reduction of vaccine hesitancy. Ottawa (ON): Canadian Immunization Conference; 2018 Dec 4.

15. MacDonald NE, Dubé E. Le Centre canadien de ressources et d'échange sur les données probantes en vaccination (CANVax) : Une nouvelle ressource permettant de résumer les données probantes sur la vaccination. Relevé des maladies transmissibles au Canada 2020;46(1):17-21. DOI

16. Centre canadien de ressources et d'échange sur les données probantes en vaccination. Ottawa (ON) : CANVax; (Accédé 2020-02-17). https://www.canvax.ca/fr

17. Paulussen TG, Hoekstra F, Lanting $\mathrm{Cl}$, Buijs GB, Hirasing RA Determinants of Dutch parents' decisions to vaccinate their child. Vaccine 2006 Jan;24(5):644-51. DOI PubMed

18. Gagneur A, Bergeron J, Gosselin V, Farrands A, Baron G. A complementary approach to the vaccination promotion continuum: an immunization-specific motivational-interview training for nurses. Vaccine 2019 May;37(20):2748-56.

DOI PubMed
19. Gagneur A, Gosselin V, Bergeron J, Farrands A, Baron G. Development of motivational interviewing skills in immunization (MISI): a questionnaire to assess MI learning, knowledge and skills for vaccination promotion. Hum Vaccin Immunother 2019;15(10):2446-52. DOl PubMed

20. Improving vaccination demand and addressing hesitancy. Geneva (CH): World Health Organization (Accédé 202002-17). https://www.who.int/immunization/programmes_ systems/vaccine_hesitancy/en/

21. L'entretien motivationnel, une stratégie efficace pour diminuer l'hésitation à la vaccination : 28 Novembre 2019 : Atelier méthodologique. Montréal (QC) : 2019. https://www. inspq.qc.ca/jasp/programmes-scientifiques

22. Vers l'acceptation : comment aborder l'hésitation envers la vaccination dans une pratique achalandée. Ottawa $(\mathrm{ON})$ : Société canadienne de pédiatrie (Accédé 2020-02-17). https://www.cps.ca/fr/reticence-vaccination 